\title{
Open Science. Kooperation zwischen Bibliothek und Wissenschaft
}

\section{Wandel der Medien}

Das Interesse am Wandel von Medien und die bisweilen ausgeprägte Bereitschaft, neue Medien auszuprobieren und zu nutzen, sind in den Möglichkeiten und Vorteilen begründet, die neue Medien gegenüber vorausgegangenen, „alten" Medien im Regelfall bieten. In diesem Zusammenhang spielen einerseits die Auffindbarkeit, Nutzbarkeit, Verbreitung und Zugänglichkeit neuer Medien eine entscheidende Rolle. Andererseits sind Authentizität, Verlässlichkeit und nachhaltige Verfügbarkeit von zentraler Bedeutung für die Inhalte, deren Verbreitung und Überlieferung von den Medien bestimmt sind. Der Übergang von der Handschrift zum Druck hat vor diesem Hintergrund vergleichbare Fragen aufgeworfen, wie sie sich heute beim Übergang von analogen zu digitalen Medien stellen. Mit dem Buchdruck haben sich über neue Zugänge zu Wissensgütern hinaus bis dahin ungekannte Möglichkeiten der Alphabetisierung aufgetan, um Wissen in schriftlicher Form rezipieren und dokumentieren zu können. Zugleich war dies mit erheblichen Auswirkungen auf gesellschaftliche, kulturelle, politische, wirtschaftliche und viele weitere Entwicklungen verbunden, die mit dem Aufkommen gedruckter Medien unmittelbar in Zusammenhang standen.

Demgegenüber hat sich mit dem Internet ein Kosmos an Informationen, Inhalten, Medien und Wissensobjekten eröffnet, der in diesem Umfang mit den in Archiven, Bibliotheken, Museen, Verlagen und anderen Intermediären vorgehaltenen, analogen Medien niemals möglich gewesen wäre. Mit der Aktualität jedweder Information und ihrer Verfügbarkeit unabhängig von Ort und Zeit sind die Vorteile digitaler Medien evident. Leser und Rezipienten, die in der Informations- und Wissensgesellschaft zu Konsumenten und Verbrauchern geworden sind, werden durch diese Vielfalt an Wissenszugängen genauso zufriedengestellt wie sie durch den Überfluss an Informationen zugleich überfordert sind. Das digitale Paradigma ist mittlerweile tief in die Alltagsrealität diffundiert und wirkt sich mit hoher Dynamik auf die damit einhergehenden Veränderungen in Arbeitswelt, Gesellschaft, Kultur, Politik, Wirtschaft und Wissenschaft aus. Davon betroffen sind in besonderer Weise diejenigen Akteure und Institutionen, die im Kontext der Produktion und Distribution von Inhalten und Wissensgütern als Intermediäre an vorderster Front des Wandels von analogen zu digitalen Medien stehen. Chancen und Risiken dieser Transformation kön-

ว Open Access. (c) 2018 Andreas Degkwitz, publiziert von De Gruyter. (๔) BY-Nc-ND Dieses Werk ist lizenziert unter der Creative Commons Attribution-NonCommercial-NoDerivatives 4.0 Lizenz. 
nen vor allem bei privatwirtschaftlich und öffentlich-rechtlich organisierten Dienstleistungsunternehmen beobachtet werden. Das Beispiel der wissenschaftlichen Bibliotheken kann dies verdeutlichen, indem - wie in anderen Servicebranchen auch - herkömmliche Geschäfts- und Organisationsmodelle zur Umsetzung des institutionellen oder unternehmerischen Auftrags in immer geringerem Umfang die Anforderungen und Veränderungen aufgreifen können, die auf der Digitalisierung beruhen.

\section{Alte und neue Servicemodelle}

Mit ihren über Jahrhunderte gepflegten Sammlungen gedruckter Bücher und Zeitschriften standen wissenschaftliche Bibliotheken bisher meistens am Anfang und Ende von Forschungsprozessen. Denn Bibliotheken wurden - und werden noch immer - im Regelfall aufgesucht oder genutzt, um den Forschungsprozess mit der Recherche nach Informationen $\mathrm{zu}$ beginnen. Für die dann abschließende Veröffentlichung der Forschungsergebnisse boten - und bieten - Bibliotheken die langfristige Speicherung und Verfügbarkeit von Publikationen im Rahmen ihres Auftrags der Informations- und Literaturversorgung. Mit unterschiedlicher Intensität und abhängig von den Fachgebieten haben die Informations- und Literaturangebote der Bibliotheken den Forschungsprozess in der Weise begleitet, dass Bibliotheken als Arbeitsräume oder im Sinne von „Laboren“ genutzt wurden und weiterhin dafür in Anspruch genommen werden.

Doch dieses Szenario ändert sich im Zuge digitaler Forschung und Lehre. Einerseits werden die herkömmlichen Aufgaben der Literatur- und Informationsversorgung mit der Bereitstellung und Nutzung von E-Books und E-Journals in digitale Services überführt. Beschaffung und Bereitstellung von E-Medien haben veränderte Arbeitsabläufe und Workflows zur Folge, die für E-Book- und E-Journal-Pakete schon realisiert sind. Andererseits ermöglichen digitale Arbeitsformen enge Kooperationen zwischen Infrastruktur und Wissenschaft, die Forschungsprozesse über generische Infrastrukturdienste hinaus unmittelbar mit fachlich spezifischen Beratungsleistungen, Infrastrukturdiensten und Werkzeugen unterstützen; dies lässt sich zum Beispiel bei Services für den Umgang mit Forschungsdaten beobachten. Zugleich ergeben sich daraus neue Anforderungen an die Serviceportfolios wissenschaftlicher Bibliotheken. Die Digitalisierung und Verarbeitbarkeit historischer und wissenschaftsrelevanter Sammlungen, die Maßnahmen im Kontext von Forschungsdaten, die Unterstützung und Weiterentwicklung von Open Access und viele weitere Dienstleistungen, die 
Nutzerinnen und Nutzer erwarten, geben dies eindeutig zu erkennen. Angesichts dessen kommen mit digitalen Arbeitsformen in Forschung, Lehre und Studium die Bibliotheken in die Situation, die veränderten Anforderungen und Erwartungen mit neuen Serviceleistungen aufzugreifen und zu realisieren. Dabei setzen die Realisierung neuer Services und das Re-Design der Portfolios voraus, dass auch die Geschäfts- und Organisationsmodelle von Bibliotheken entsprechend angepasst und verändert werden. ${ }^{1}$ Diese Herausforderung wirft die Frage auf, in welche Richtung sich wissenschaftliche Bibliotheken im Kontext digitaler Wissenschaften weiterentwickeln und wie sich die Kooperationen mit Forschenden, Lehrenden und Studierenden sowie mit anderen bibliothekarischen Einrichtungen darstellen werden.

\section{Open Science}

Dass Informationstechnik und Informationswissenschaft wesentliche Voraussetzungen für digitale Arbeitsumgebungen und Infrastrukturen sind, steht außer Frage. Digitalisierung jedoch auf die technische Realisierung von Infrastrukturen und Services zu beschränken, blendet wesentliche Aspekte der damit verbundenen gesellschaftlichen, kulturellen und politischen Veränderungen aus. Auch für den Wissenschaftsbereich lassen sich über die technologiegetriebenen Grundlagen der Digitalisierung hinaus Einflussfaktoren identifizieren, die die bestehenden Arbeits- und Fachkulturen aller Wissenschaftsdisziplinen verändern. Dabei stehen der Anspruch und die Herausforderung einer „offenen Wissenschaftspraxis“ im Mittelpunkt. Was wird in diesem Zusammenhang unter „offen“ verstanden und was ist das Ziel von Open Science? Ist Wissenschaft nicht schon immer „offen“ gewesen, wie der hohe Stellenwert des wissenschaftlichen Publizierens für die Reputation von Wissenschaftlerinnen und Wissenschaftlern zeigt? Antworten darauf geben die im europäischen Kontext etablierten Initiativen wie beispielsweise die Open Science Policy Platform (OSPP) $)^{2}$ oder der Open Science Monitor.

Der Open Science Monitor als Instrument zur Prüfung der Offenheit von Wissenschaft verdeutlicht die Anforderungen, die sich auf der Ebene der europäischen Förder- und Wissenschaftspolitik mit Open Science verbinden, anhand zentraler Elemente des Forschungsprozesses: Open Research Data, Open

1 Vgl. Degkwitz, Andreas: Überholtes Geschäftsmodell? Bibliotheken in der digitalen Transformation. In: Forschung und Lehre 23 (2016) H. 9. S. 770-772.

$2 \mathrm{https}$ ://ec.europa.eu/research/openscience/index.cfm?pg=open-science-policy-platform. Alle aufgeführten Internetressourcen wurden zuletzt am 1. Dezember 2017 aufgerufen. 
Scholarly Communication, Open Access to Publications. ${ }^{3}$ Denn Open Access, Open Data, Open Source und Open Clouds sollen sich eng an den so genannten FAIR-Prinzipien orientieren und nach diesen Prinzipien realisiert werden. ${ }^{4}$ Werden Forschungsprozesse und Forschungsergebnisse „FAIR“ nach den Kriterien „Findable“, „Accessible“, „Interoperable“ und „Re-usable“ und der darauf aufsetzenden Standards gestaltet, werden die Kollaborationspotenziale des Internets besser ausgeschöpft und der Informations- und Wissenstransfer nachhaltig verbessert. Zugleich können auf diese Weise die dringend erforderlichen Voraussetzungen für Nachvollziehbarkeit und Transparenz von Forschungsergebnissen in einem deutlich größeren Umfang erfüllt werden als bisher.

Wie ordnen sich die wissenschaftlichen Bibliotheken in den Kontext von Open Science und in das Open Science zugrunde liegende neue Eco-System der Wissenschaft ein? In erster Annäherung liegt als Vermutung nahe, dass Bibliotheken sich evolutionär in dieses Szenario integrieren, ohne sich dabei in der Situation zu sehen, ihr Geschäfts- und Servicemodell grundsätzlich zu verändern. Doch diese Sicht auf die bibliothekarische Weiterentwicklung erweist sich als $\mathrm{zu}$ intrinsisch, denn die ökonomischen und organisatorischen Auswirkungen des neuen Eco-Systems, das sich mit der Etablierung von Open Science verbindet, bleiben dabei weitgehend unberücksichtigt, wie sich am Beispiel des Open-Access-Publizierens aufzeigen lässt.

\section{Wandel durch Open Access}

Mit Open Access wandelt sich das Finanzierungsmodell des wissenschaftlichen Publizierens und der Informations- und Medienversorgung, indem die damit verbundenen Kosten nicht mehr nutzerbasiert auf der Grundlage von Subskriptionen, sondern publikationsbasiert auf der Grundlage von Artikelgebühren gedeckt werden. Wenn sich dieses Modell in der längerfristigen Perspektive zum Standard entwickelt, ist absehbar, dass die Mittel für die Finanzierung der Article Processing Charges (APCs) nicht mehr von den Bibliotheken, sondern von den Wissenschaftlerinnen und Wissenschaftlern als Autorinnen und Autoren bewirtschaftet und verausgabt werden. Selbstverständlich werden die Bibliotheken die Autorinnen und Autoren dabei beraten und unterstützen. Doch die bisherige Verantwortung der Bibliotheken für das Erwerbungsbudget wird aller Voraussicht nach nicht fortbestehen, sobald sich Open Access auf der Basis von

3 https://ec.europa.eu/research/openscience/index.cfm?pg=home\&section=monitor.

$4 \mathrm{http} / /$ www.forschungsdaten.org/index.php/FAIR_data_principles. 
APCs zum führenden Finanzierungsmodell der Informations- und Medienversorgung entwickelt hat. Damit wird eine zentrale Komponente des Auftrags der Bibliotheken an die Wissenschaft übergeben.

Darüber hinaus verändert sich mit den unterschiedlichen Modellen des Open-Access-Publizierens die Kooperation zwischen Bibliotheken und Wissenschaft. Verlegerische Initiativen werden zunehmend von Fachcommunitys oder auch von Universitäten aufgegriffen. Universitätsverlage, die im Regelfall Bibliotheken zugeordnet sind, haben sich - auch in Kooperationen auf regionaler Ebene - zu Publikationsdienstleistern an zahlreichen deutschen Bibliotheken entwickelt. Aus diesen Initiativen heraus ergibt sich zudem die Möglichkeit, neue Publikationsformen auszuprobieren und zu testen, um z. B. Forschungsergebnisse aus digitalen Arbeitskontexten und virtuellen Forschungsumgebungen zur Publikation bringen zu können. Weiterhin werden mit der Auswertung, Evaluation und Nachnutzung von Metadaten neue Services in die Serviceportfolios von Bibliotheken etabliert. Dabei geht es um Identifizierung und Ranking des Publikationsoutputs einzelner Wissenschaftlerinnen und Wissenschaftler oder von Forschungseinrichtungen und Hochschulen. Diese Dienste können in einem engen Zusammenhang mit den von den Hochschulen betriebenen Forschungsinformationssystemen stehen. Schließlich kann durch die Verlinkung bibliografischer Metadaten und Autoren-Identifikationen wie ORCID ${ }^{5}$ verstärkt zur fachlichen und institutionellen Kontextualisierung, Sichtbarkeit und Vernetzung von Forschungsprojekten und Forschungsergebnissen beigetragen werden. Auch dabei geht es um Entwicklungen, die im flächendeckenden Umfang erst mit der Digitalisierung möglich sind und zu größerer Offenheit von Forschungsaktivitäten sowie zur Verbesserung des Informations- und Wissenstransfers führen.

\section{Neues Eco-System}

Mit diesem Wandel in Forschung, Lehre und Studium erfolgt der Eintritt in ein Eco-System, das neue Geschäfts- und Servicemodelle etabliert und das Selbstverständnis der daran beteiligten Akteure und Institutionen - nicht akzidentiell, sondern im Grundsatz - verändert. Wohin entwickelt sich dieses Szenario insgesamt? Und wie gestaltet sich der Entwicklungsprozess? Diese Fragen machen erneut deutlich, dass die Auswirkungen der Digitalisierung über den Aufund Ausbau technischer Infrastrukturen und Services weit hinausreichen. Ab-

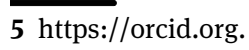


schließende Antworten darauf hängen wesentlich von fachwissenschaftlich orientierten, gesellschaftlichen und politischen Einflussfaktoren ab. Deren Gewicht und Tragweite sind aktuell nur in Ansätzen identifizierbar und erfordern zu ihrer Einordnung weitere Analysen und Auswertungen.

In diesem Zusammenhang erweist sich die kürzlich erschienene Studie Knowledge Exchange approach towards Open Scholarship als sehr aufschlussreich. ${ }^{6}$ In dieser wird auf die noch offenen und ungeklärten Themen eingegangen, doch zunächst nur das Spektrum der absehbar klärungsbedürftigen Fragestellungen dargelegt. Dazu gehören vor allem aus ökonomischer Sicht: ${ }^{7}$

- die Bewertung akademischer Güter (,goods“) aus der Perspektive der Politik, der Fachcommunitys und der einzelnen Wissenschaftlerinnen und Wissenschaftler

- die Anreizmechanismen, die wissenschaftliches Engagement und Reputationsbildung beeinflussen und steigern

- die Notwendigkeit von Governance-Strukturen zur Unterstützung und Finanzierung von Open Scholarship

- die Funding-Optionen für den Auf- und Ausbau und für den Betrieb von Infrastrukturen

- der Kontrollverlust, der sich aus privatwirtschaftlichen Marktmonopolen ergibt und Open Scholarship beeinträchtigt

Diese und weitere Aspekte lassen erkennen, bei welchen Themen sich Open Science auf das bestehende Eco-System der Wissenschaft absehbar auswirken wird. Dass es dabei um einen tief greifenden - durchaus auch disruptiven Wandel geht, ist evident.

\section{Künftige Rolle von Bibliotheken}

Der Wandel von Medien ist für Bibliotheken nicht Neues. Wie beim Übergang vom Pergament zum Papier erweist sich die bit- und bytebasierte Transformation der Medien als gravierend und folgenreich. $\mathrm{Zu}$ den Herausforderungen für Bibliotheken gehört in diesem Zusammenhang einerseits die Mitwirkung an der Entwicklung von Standards, die die Auffindbarkeit und Nutzung

6 Vgl. Knowledge Exchange approach towards Open Scholarship. Based on contributions from the Knowledge Exchange Open Scholarship Advisory Group and edited by Cameron Neylon, August 2017. https://repository.jisc.ac.uk/6685/1/KE_APPROACH_TOWARDS_OPEN_SCHOLARSHIP_AUG_2017.pdf.

7 Vgl. Knowledge Exchange (wie Anm. 6), S. 24-32. 
der neuen Medien sicherstellen. Andererseits müssen die Geschäfts- und Servicemodelle der Bibliotheken die Veränderungen aufgreifen, die sich aus der digitalen Transformation ergeben. Für die Entwicklung neuer Standards erweist sich Open Access erneut als ein gutes Beispiel, denn um den Anforderungen für wissenschaftliche Publikationen zu entsprechen, müssen Open-Access-Publikationen auffindbar sein sowie gelesen und genutzt werden können und nachhaltig verfügbar sein. Diese Anforderungen werden in der Weise umgesetzt,

- dass Open-Access-Veröffentlichungen nach gängigen Metadatenstandards erschlossen werden und damit auffindbar sind,

- dass sie mit unterschiedlichen Präsentationsformaten wie PDF oder HTML rezipiert und genutzt werden können

- und dass sie schließlich auf Repositorien gespeichert werden und dort dauerhaft zur Verfügung stehen.

Mit dem Einsatz von Standards und Standardverfahren wird Offenheit nicht nur im Sinne der freien Zugänglichkeit - auf Basis entsprechender Creative-Commons-Lizenzen - sichergestellt, sondern vor allem die Nutzbarkeit und Verfügbarkeit von Open-Access-Publikationen auf Dauer gewährleistet. ${ }^{8}$ Werden diese Standards nicht eingesetzt, besteht das hohe Risiko, dass Open-Access-Veröffentlichungen nur zufällig identifizierbar und weder dauerhaft verfügbar noch nutzbar sind. Damit würden sie dem weiterhin geltenden Nachhaltigkeitsanspruch an wissenschaftliche Veröffentlichungen nicht genügen. Insofern erweisen sich die Standards ganz eindeutig als unabdingbare Voraussetzung für die mit Open-Access-Publikationen angestrebte Zugänglichkeit von Forschungsergebnissen.

Doch die künftige Rolle von Bibliotheken im Kontext von Open Science ist nicht auf Open Access beschränkt. Die besondere Qualität digitaler Arbeitsformen und Herangehensweisen liegt in den Potenzialen der dynamischen Verarbeitung und Vernetzung von digitalen Objekten wie Texten, Bildern und weiteren Datenbeständen. Die Zahl der dafür zur Verfügung stehenden Materialien wächst stetig durch die Digitalisierung historischer Bestände und Born-DigitalMaterialien. Insofern ist künftig von einer erheblichen Zunahme digitaler Forschungsaktivitäten - auch in den Geistes- und Kulturwissenschaften - auszugehen.

Diese Entwicklung hat zur Folge, dass digitale Inhalte und Ressourcen nicht mehr nach traditionellem Verständnis als „lokal“ vorgehaltene Bibliotheksbestände gesammelt, erschlossen und zur Verfügung gestellt werden. Viel-

8 Vgl. Degkwitz, Andreas: „Open Science“ - Treiber des digitalen Wandels in Bibliotheken. In: die hochschule 26 (2017) H. 2. S. 20-29. 
mehr und darüber hinaus müssen digitale Inhalte und Objekte für ihre Nutzung und Nutzbarkeit in Forschungskontexten von Bibliotheken aufbereitet und kuratiert werden. Damit kommen Bibliotheken zunehmend in die Situation, zur Bearbeitung und Nutzung digitaler Objekte passende Dienste und Werkzeuge zur Verfügung zu stellen und aktiv zu vermitteln; dies ergibt sich aus der Kooperation mit den Zielgruppen und deren Erwartungen. Aus diesem Zusammenhang entstehen neue datentechnische Anforderungen an die Aufbereitung und Verfügbarmachung von Metadatenstrukturen für solche Objekte, die eine dynamische Verarbeitung auch im Sinne von Big-Data-Analytik implizieren. Angesichts der Entwicklungen in der digitalen Wissenschaft ist in dieser Hinsicht eine erhöhte Nachfrage der Forschenden zu erwarten. Mehr denn je zeigt sich die Bibliothek als eine intermediäre Einrichtung herausgefordert, unterschiedliche Anforderungen zu möglichst leicht nutzbaren Dienstleistungen zu bündeln und $\mathrm{zu}$ vermitteln.

Zugleich erweisen sich Kooperationen zwischen Bibliotheken und entsprechend spezialisierten Anbietern wie Infrastrukturanbieter oder Kompetenzzentren für unterschiedliche Fachgebiete als ebenso hilfreich wie notwendig, denn digitale Forschung ist an vielen Stellen zu komplex, um allein über lokale Angebote ausreichend adressiert werden $\mathrm{zu}$ können. Je spezialisierter und anspruchsvoller die Forschungskontexte sind, desto mehr verschiebt sich die Rolle der Einrichtung vom Anbieter zum Vermittler. Die Kompetenz der Bibliothek liegt hier im Überblickswissen und potenziell in einem etablierten Expertennetzwerk, das lokal auftretende Anfragen bedarfsgerecht an spezialisierte Ansprechpartner weitergibt.

\section{Zusammenfassung}

Wissenschaftliche Bibliotheken können eine Schlüsselposition bei der Unterstützung von Open Science und den mit Open Science verbundenen Entwicklungen in Forschung und Lehre ihrer Einrichtung einnehmen. Die Vermittlung von Inhalten und Wissensressourcen wird dabei um die aktive Vermittlung von Infrastrukturangeboten, Informations- und Medienkompetenzen, Methoden, Werkzeugen bis hin zur Vermittlung spezialisierter Kooperationspartner ergänzt. Auf diese Weise wird die Trennung zwischen Informationsversorgung, Durchführung von Forschung und Kommunikation von Ergebnissen aufgehoben, was einen zentralen Effekt von Open Science darstellt. Die Bibliotheken werden zu Partnern der Wissenschaft über alle Stufen des Forschungsprozesses hinweg. 
Das primär auf bestandsbezogene Bereitstellung und Erschließung fokussierende, traditionelle Servicemodell wissenschaftlicher Bibliotheken genügt nicht, um datenintensive und toolbasierte Arbeits- und Forschungsformen organisatorisch und technisch in der notwendigen fachlichen Breite zu unterstützen. Vielmehr müssen Organisations- und Servicemodelle entwickelt werden, die zur direkten Kollaboration zwischen Bibliothek und Wissenschaft beitragen und damit die Partizipation an Wissenstransfer und Kompetenzentwicklung verbessern. Dabei werden Bibliotheken als Scholarly Makerspaces ${ }^{9}$ verstanden, die digitale Arbeitsformen in Forschung und Lehre mit entsprechenden Infrastruktur- und Serviceangeboten ermöglichen und dabei Infrastrukturdienste und Werkzeuge unmittelbar integrieren.

9 Vgl. Degkwitz, Andreas: The Interactive Library as a Virtual Working Space. In: LIBER Quarterly 27 (2017) H. 1. S. 127-137. https://www.liberquarterly.eu/article/10.18352/lq.10214/. 